\title{
Wing/kite-based wind energy generation: An overview
}

\author{
M. Milanese \\ Kitenergy S.r.l. - Torino, Italy
}

\begin{abstract}
Summary. - Several technologies, aimed at converting high-altitude wind into electricity, are actually being investigated by companies, research centers and universities worldwide, and the community of people working in this field has coined the term airborne wind energy (AWE) as a common umbrella for these concepts. Indeed, many basic ideas that are now being developed in the context of AWE were already present in patents and publications since the '70s. Then, these ideas remained somehow silent, until more recent years, when several research groups and companies started to carry out theoretical, numerical and experimental analyses, made possible by important advances in diverse fields like materials, aerodynamics, sensors, computation and control. In this lecture, the basic AWE concepts and results that have been up to date accomplished are overviewed, with a focus on a particular class of AWE generators, namely with flexible wings and ground level generators, and emphasis on optimization and control aspects. Finally, we delineate what challenges are still to be faced, in order to fully demonstrate the viability of airborne wind energy.
\end{abstract}

The full text of this contribution was not received.

The slides of the presentation are available at http://static.sif.it:8080/SIF/resources/public/files/en2012/Milanese_Slides.pdf 